\title{
A Teoria Piagetiana das Trocas Sociais e sua Aplicação aos Ambientes de Ensino-aprendizagem
}

AntÙnio Carlos da Rocha Costa

\section{The Piagetian Theory of Social Exchanges and its Application to Learning Environments}


Resumo: Este artigo resume a teoria piagetiana das trocas sociais, apresenta o $n^{\circ}$ cleo formal da teoria ó a teoria dos valores de troca qualitativos ó e mostra algumas possibilidades de utilizaÁ „, o dessa teoria de valores de troca no contexto da Inform·tica na EducaÁ,o.

Palavras-chave: Inform·tica na educaÁ,,o. Teoria piagetiana das trocas sociais.

Abstract: This paper summarizes the piagetian theory of social exchanges, presents its formal nucleus ó the theory of qualitative exchange values ó, and shows some possible uses of the theory of exchange values in the context of Educational Informatics.

Keywords: Computers in education. Piagetian theory of social exchanges.

COSTA, AntÙnio Carlos da Rocha. A Teoria Piagetiana das Trocas Sociais e sua AplicaÁ,,o aos Ambientes de Ensino-aprendizagem. Informática na Educação: teoria \& prática, Porto Alegre, v. 6, n. 2, p. 77-90, jul./dez. 2003. 


\section{Introdução}

O presente artigo procura sumariar a sociologia piagetiana. Inicialmente demonstra-se de que os aspectos sociais das relaÁres humanas $\mathrm{n}$,o foram desconsiderados nem menosprezados por Piaget, na elaboraÁ,,o de sua teoria do desenvolvimento cognitivo. Na seq, Íncia tem-se um resumo da sociologia piagetiana, apresentando suas noÁıes centrais, quais sejam, as noÁıes de totalidade social e de trocas sociais. Apresena-se o $n$ ' cleo formal dessa sociologia, que È a teoria dos valores de troca qualitativos, e a aplicaÁ,,o, por Piaget, desta teoria na formalizaÁ,,o dos aspectos morais e jurl̀dicos das trocas sociais.

AlÈm disso, o artigo mostra a import,ncia da teoria sociolÛgica de Piaget para a Inform·tica na EducaÁ,,o.Descreve uma aplicaÁ,o computacional da teoria dos valores de troca na -rea da Inteligíncia Artificial Distribulda (COSTA, 2002; RIBEIRO, 2003a; RIBEIRO, 2003b), propondo o uso dessa teoria para a modelagem dos aspectos morais e jurl̀dicos das trocas entre agentes de um sistema multiagentes. Introduz uma delimitaÁ,,o das duas principais formas de utilizaÁ,,o da teoria dos valores de troca em ambientes computacionais de apoio ao ensinoaprendizagem, quais sejam, a modelagem das conversaÁıes (aÁıes ling, Ìsticas) e a an·lise das trocas realizadas por aÁıes materiais. Apresenta um detalhamento preliminar da quest, ,o que d. sentido 'Itimo $\neq$ teoria dos valores de troca, que È a quest, ,o do uso dos sistemas de valores como instrumentos da manutenÁ,,o do equilìbrio normativo das trocas sociais, e uma proposiÁ,,O inicial de um mecanismo de regulaÁ,,O desse equilìbrio no contexto dos ambientes computacionais.

\section{O pensamento sociológico de Piaget}

O pensamento sociolÛgico de Piaget se concentra refletido em uns poucos textos fundamentais. Estes textos est, ,o compilados no livro intitulado Études Sociologiques (PIAGET, 1965), que tem traduÁ,,o brasileira (PIAGET, 1973b) da $1^{\text {a }}$ ediÁ,,o e inglesa (PIAGET, 1965b), da 2a ediÁ,„o.

Os textos principais s,,o os seguintes:

¿o quadro geral da concepÁ,,o sociolûgica de Piaget aparece no capìtulo ìA ExplicaÁ,,o em Sociologiaî (PIAGET, 1973c), que faz parte do terceiro tomo de sua principal obra epistemolÛgica, a Introdução à Epistemologia Genética (PIAGET, 1950); o capltulo apresenta o pensamento epistemolûgico de Piaget sobre a Sociologia e inclui os resultados obtidos nos seus trabalhos anteriores, que est,„o listados a seguir;

$\Sigma$ o artigo ìLÛgica GenÈtica e Sociologiaî (PIAGET, 1995a), publicado originalmente em 1928, que È uma primeira abordagem $\ddagger$ quest,„o das relaÁıes entre LÛgica e Sociologia, consolidando a noÁ,,o piagetiana de totalidade social;

$\sum 0$ artigo ìEnsaio sobre a Teoria dos Valores Qualitativos em Sociologia Est.tica (îSincrÙnicaî)î (PIAGET, 1973g), publicado originalmente em 1941, Piaget desenvolve a teoria dos valores de troca, que È o n cleo formal de sua teoria das trocas sociais e que consolida as relaÁıes dessa teoria com a noÁ,,o de totalidade social, incluindo os aspectos morais e jurlidicos dessa totalidade;

¿o artigo ìAs RelaÁıes entre a Moral e o Direitoî (PIAGET, 1973f), publicado originalmente em 1944, que explicita mecanismos 
operatÛrios comuns ao funcionamento das regras morais e das regras jurldicas;

$\Sigma$ o artigo ìAs OperaÁıes LÛgicas e a Vida Socialî(PIAGET, 1973f), publicado originalmente em 1945, que explicita a presenÁa das estruturas de grupamentos nas trocas sociais.

Que Piaget abstraiu as trocas sociais na maioria dos seus estudos da Psicologia da Inteligíncia È um fato ineg·vel. Que desconheceu ou negou a import,ncia dessas trocas sociais para o desenvolvimento cognitivo da crianÁa È simplesmente uma falsidade.

Em v rias ocasiles, Piaget manifestou explicitamente a dependíncia do desenvolvimento cognitivo em relaÁ,,o aos fatores sociais:

O conhecimento humano Ė essencialmente coletivo e a vida social constitui um dos fatores essenciais da formaÁ,,o e do crescimento dos conhecimentos prË-cientìficos e cientìficos.í (PIAGET, 1973c, p.17)

O conjunto das condutas humanas comporta, desde o nascimento e em graus diversos, um aspecto mental e um aspecto social.í (PIAGET, 1973c, p. 21)

O desenvolvimento individual È em parte condicionado pelo meio social, e a psicogínese È parcialmente uma sociogínese. (PIAGET, 1973c, p. 27)

Mas, se a sociogínese das noÁıes intervĖm, pois, na psicogínese, desde os es$t \cdot g i o s$ elementares do desenvolvimento, $\dot{E}$ IÛgico que sua influíncia cresce em progress,,O geomĖtrica em relaÁ,,O ‡ suces$\mathrm{s}$, , O dos est-gios ulteriores.í (PIAGET, 1973c, p. 29)

A construÁ,o progressiva das operaÁıes intelectuais supıe uma interdependíncia crescente ente os fatores mentais e as interaÁıes interindividuais. Uma vez as operaÁıes constituldas, um equilìbrio se estabelece entre o mental e o social, no sentido de que o indivìduo tornado membro adulto da sociedade $n$, o poderia mais pensar fora desta socializaÁ,o acabada.í (PIAGET, 1973c, p. 29)

Por que, ent,,o, Piaget teria abstral̀do a referíncia aos fatores sociais na esmagadora maioria dos seus trabalhos sobre o desenvolvimento da inteligíncia na crianÁa?

\begin{abstract}
A interdependíncia da sociogínese e da psicogínese se faz sentir de forma particularmente marcante no terreno da psicologia da crianÁa, ‡ qual fizemos numerosos apelos para explicar a construÁ,,o das noÁıes. Ora, tal recurso ao desenvolvimento intelectual da crianÁa, concebido a tìtulo de embriogínese mental, È um recurso do qual $\mathrm{j}$ - defendemos o princlipio ${ }^{1}$ apelando para os serviÁos prestados pela embriologia biolÛgica ‡ anatomia comparada, pÙde deixar certo mal-estar no espìrito de mais de um leitor. A psicologia da crianÁa explicaria sem d' vida o modo de formaÁ,,o das noÁıes ou das operaÁıes, objetariam alguns leitores, ${ }^{2}$ se a crianÁa pudesse ser estudada em si mesma, independentemente de qualquer influíncia adulta, e se ela construlısse assim seu pensamento sem se servir dos elementos essenciais no meio social. (PIAGET, 1973c, p.26)
\end{abstract}

PorÈm, continua Piaget:

Mas o que È a crianÁa em si mesma, e as crianÁas $\mathrm{n}, 0$ existem a $\mathrm{n}, 0$ ser em relaÁ,OO a certos meios coletivos bem determinados? ëlsso È evidente, e, se se chama ìpsicologia da crianÁaî ao estudo do desenvolvimento mental individual, Ėsimplesmente em referíncia aos mÈtodos experimentais utilizados nessa disciplina: na verdade, e tanto no que concerne łs noÁres explicativas da qual se serve, quanto relativamente a seu objeto de investigaÁ,,O, a psicologia da crianÁa constitui setor da sociologia, consagrado ao estudo da socializaÁ,,o do indivilduo, ao mesmo tempo em que se constitui setor da psicologia mesma. (PIAGET, 1973c, p.26)

1 A traduÁ,o brasileira traz ìproibimosíi ao invĖs de ìdefendemosíi! (cf. PIAGET, 1995b, p.35).

2 A traduÁ,o brasileira $n$, o traz essa cl-usula de relativizaÁ,,o (cf. PIAGET, 1995b, p.35). 
Ou seja, a abstraÁ,,o dos fatores sociais no estudo do desenvolvimento da crianÁa È uma quest,,o de mÈtodo de pesquisa, uma opÁ,o metodolÛgica durante o processo de investigaÁ,,o, n„,o uma quest, ,o de fundamento ou de posicionamento teÛrico sobre o papel da vida social naquele desenvolvimento. Como tal, essa abstraÁ,,o deve ser feita de modo a ter a menor influíncia possìvel sobre os resultados obtidos. Assim, logo que os resultados procurados forem encontrados, importa imediatamente reintegrar os fatores sociais $\ddagger$ an.lise, para que aqueles resultados possam ser retrabalhados $\ddagger$ luz dessa perspectiva ampliada: ìRenunciemos agora ao artiflycio que nos fez considerar como um sistema fechado o indivìduo, assim como suas relaÁıes com o meio fìsico, e perguntemos o que ser „o as relaÁıes de ordem intelectual com os outros indivìduosî (PIAGET, 1973e, p.178).

A quest,,o da explicaÁ,,o sociolûgica do desenvolvimento cognitivo se impıe como necess·ria, ent,„o, para complementar a explicaÁ,„o biolÛgica e psicolÛgica:

O problema È ent,,O o seguinte: se a IÛgica consiste numa organizaÁ,,o de operaÁles ques,„o definitivamente interiorizadas e tornadas reversìveis, pode-se conceber que o indivìduo consegue atingir sozinho esta organizaÁ,,o, ou a intervenÁ,,o de fatores interindividuais È necess ria para explicar o desenvolvimento que acabamos de descrever? (PIAGET, 1973e, p. 178).

O resto do artigo, È claro, consiste na demonstraÁ,,o positiva de que È a segunda alternativa que È v·lida.

\section{A sociologia de pequenos grupos de Piaget}

N,,o È possìvel resumir, aqui, a sociologia de pequenos grupos que Piaget desen- volve para definir o conjunto de fatores interindividuais aos quais o desenvolvimento da inteligíncia e dos conhecimentos deve ser relacionado.

Vamos apontar as principais noÁıes dessa teoria sociolûgica, enfatizando o seu n' cleo operatÛrio, o qual cremos poder usar como base para fundamentar o trabalho relativo aos aspectos morais das interaÁıes em sistemas cooperativos de apoio ao ensino-aprendizagem.

As trís noÁıes centrais S,O: a noÁ,,O de totalidade social, que orienta todo o processo de estruturaÁ,,o da teoria; a noÁ,,o de valor de troca qualitativo, que permite captar nas trocas sociais os aspectos que $\mathrm{n}, 0$ s,,o estritamente econÙmicos; e a noÁ,,o de equilìbrio do sistema de trocas qualitativas, em funÁ,,o do qual se constituem as normas morais e jurl̀dicas.

\subsection{A noção piagetiana de totalidade social}

Contrapondo-se ao atomismo e ao puro holismo, Piaget coloca no estruturalismo relacional a soluÁ,,o para o problema da definiÁ,,o da noÁ,,o de totalidade social. A totalidade social n,o $E$ um agregado de indivìduos nem È uma realidade existente (ou prË-existente) num plano acima do plano dos indivìduos.

Podem-se reduzir, parece, todos os ìfatos sociaisî a interaÁıes entre indivìduos e, mais precisamente, łs interaÁıes que modificam o indivìduo de maneira dur vel. (PIAGET, 1973g, p.114)

As totalidades sociais oscilam entre dois tipos: num dos extremos, as interaÁıes em jogo s, o relativamente regulares, polarizadas por normas ou obrigaÁıes permanentes, e constituem sistemas compostos dos quais pressentimos a analogia com os agrupamentos operatÛrios no caso em que 
estes se aplicariam ¥s trocas e ¥s aÁıes hierarquizadas inter-individuais e $\neq s$ operaÁıes intra-individuais. No outro extremo, a totalidade constitui uma associaÁ,,o de interaÁıes interferindo entre elas e cujas formas de composiÁ,,o lembram as regulaÁıes ou os ritmos da aÁ,,o individual. (PIAGET, 1973c, p.43)

O primeiro tipo È o dos pequenos grupos, no qual as contribuiÁıes dos indivìduos ao grupo podem ser plenamente identificadas e as regras permanecem est $\cdot$ veis durante o processo de interaÁ,,O. O segundo caso È o das massas sociais. Os ambientes de ensino-aprendizagem nos quais estamos interessados $\mathrm{s}, \mathrm{O}$, evidentemente, do primeiro tipo. ${ }^{3}$

\subsection{A teoria dos valores de troca quali- tativos}

O cerne operatÛrio da sociologia piagetiana È uma teoria de valores de troca qualitativos, na qual as regras sociais (normas e leis) servem ₹ finalidade de determinar e manter o equilìbrio moral (e econÙmico) das trocas sociais que ocorrem no sistema.

\subsubsection{Valores e trocas}

Piaget d. uma dupla definiÁ,,o da noÁ,,o de valor. Por um lado, valor È ìqualquer coisa que dí lugar a uma trocaî. Por outro lado, Piaget define como valores os construtos mentais de car.ter qualitativo, que se associam mentalmente, no momento de uma troca, aos elementos que s,o valores no primeiro sentido e que servem ao propÛsito de avaliar esses elementos.

Os valores $\mathrm{s}, \mathrm{O}$ caracterizados como elementos qualitativos, porque deles $n_{\text {, }}, \mathrm{se}$ exige mais do que uma estrutura extensiva de ordem total, sem car·ter mÈtrico, que satisfaÁa ‡ exigĺncia mìnima de permitir que seja definida uma relaÁ,,o assimÈtrica de maior ou menor ñ ver os conceitos de quantidade, no livro Ensaio de Lógica Operatória. (PIAGET, 1973d)

Quanto $\ddagger$ noÁ„,o de troca, para simplificar o problema e permitir-lhe uma formalizaÁ,,o operatÛria, Piaget restringiu-se $\ddagger$ quest,, (na verdade, bastante geral) da troca de serviços entre sujeitos. Assim, Piaget define troca como qualquer seq, Íncia de aÁıes entre dois sujeitos, tal que um deles, pela realizaÁ,o de suas aÁıes, preste um serviço ao outro.

\subsubsection{Regras}

O terceiro conceito operatÛrio fundamental na teoria piagetiana das trocas È o conceito de regra ou norma.

As regras sociais $\mathrm{s}$, 0 pensadas com duas formas possìveis:

¿equações que devem verificar-se entre os valores envolvidos nas trocas entre indivìduos, determinando condições de equilíbrio de valores de troca;

¿operações de negaÁ,,o (puniÁıes) ou reciprocidade (reparaÁıs), que podem ser realizadas para que o equilìbrio seja restabelecido, quando ele È quebrado.

Ou seja, a teoria piagetiana das trocas sociais È uma teoria na qual a funÁ,,O reguladora das regras e das normas $\dot{E}$ estabelecida a partir de um cálculo de valores qualitativos, em que cada troca deve implicar a validade de certas equaÁıes entre os valores nela envolvidos, assim como pode

3 Note-se que Piaget (1973g) se atĖm ‡ sociologia îsincrÙnicaíi, isto È, n„o trata da evoluÁ„,o das estruturas sociais. 
implicar a realizaÁ,o de operaÁıes reguladoras, no caso de desequilìbrios.

\section{$4 \quad$ Um modo de aplicar a teoria dos valores de troca a sistemas computacionais}

Esta seÁ,o resume a teoria piagetiana das trocas sociais e sumariza nossa proposta metodolÛgica para a aplicaÁ,,o dessa teoria a sistemas computacionais em geral (COSTA, 2002).

\subsection{Modelos computacionais básicos de processos de trocas}

Os dois modelos computacionais b-sicos para processos de trocas $\mathrm{s}_{\text {, }}, \mathrm{respec}-$ tivamente denominados modelo de troca produtor-consumidore modelo de troca cliente-servidor. $\mathrm{S}$, ,o modelos cl-ssicos, tradicionalmente usados em v rias ·reas da computaÁ,o, como sistemas operacionais, bancos de dados e redes de computadores, para modelar processos de troca de serviÁos. Esses dois modelos de troca tím papel importante no modo de aplicaÁ,,o da teoria piagetiana dos valores de troca qualitativos que estamos definindo.

Nesta subseÁ,o, descrevemos esses modelos em seu conjunto e deixamos para a prÛxima subseÁ,,o a definiÁ,,o precisa do nosso modo de aplicaÁ,,o da noÁ,,o de tro$c a$, porque nos apoiaremos para isso na combinaÁ,,o dos conceitos piagetianos com os modelos de trocas computacionais resumidos aqui.

Uma troca que se d· entre dois agentes (usu.rios ou programas) em um sistema computacional, seguindo o modelo produtorconsumidor, ocorre conforme mostrado no diagrama de seq, Íncia do tipo Prd-Cns, na Figura 1. Nesse modelo, Prd Ė o agente produtor, Cns È o agente consumidor, prod Ė a aÁ,,o de produÁ,,o realizada por Prd, cons È a aÁ,,o de consumo realizada por Cns, e o processo de troca È essa seq, Íncia de operaÁıes. Trata-se de um modelo muito simples, e por isso mesmo de ampla utilizaÁ,,o.
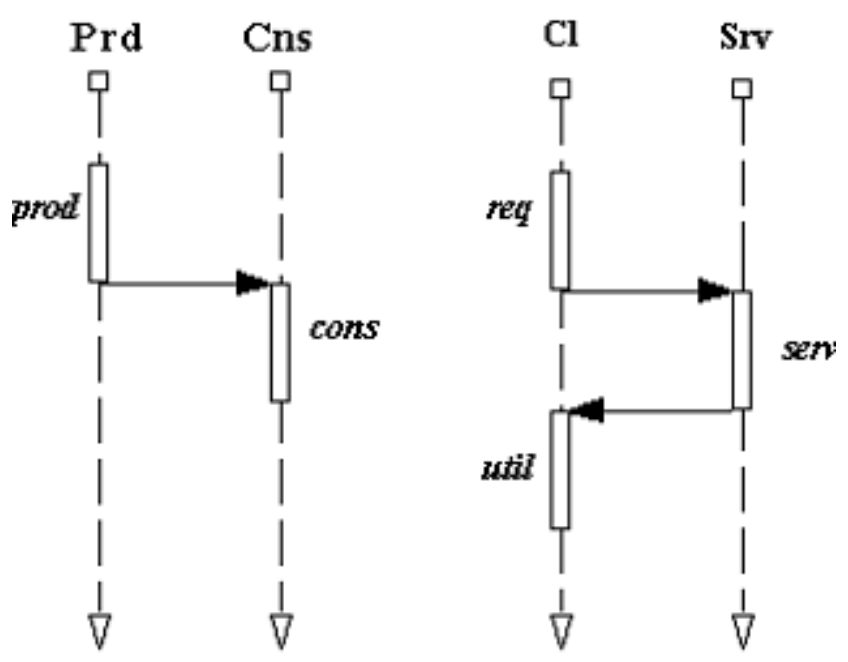

Figura 1 - Modelos computacionais b-sicos de processos de troca 
Uma troca que se d- entre dois agentes (usu.rios ou programas) em um sistema computacional, seguindo o modelo clienteservidor, ocorre conforme mostrado no diagrama de seq, Íncia do tipo $\mathrm{Cl}-\mathrm{Srv}$, na Figura 1. Nesse modelo, C/È o agente cliente, SrV È o agente servidor, req È a aÁ,,o de requisiÁ,o de serviÁo que Cl faz a Srv, serv È a execuÁ,,o do serviÁo por parte de Srv, útilE a aÁ,,o de $C /$ de utilizar os resultados do serviÁo prestado por Srv, e o processo de troca È essa seq, Íncia de operaÁıes. TambÈm se trata de um modelo simples, porÈm com utilizaÁ,,o mais especìfica em funÁ,,o de seu maior detalhamento.

\subsection{Um modo de aplicar os valores de tro- ca qualitativos às trocas em ambientes computacionais}

A partir da descriÁ,o dos tipos de trocas analisados por Piaget (1973b), È possì- vel definir um modo de aplicaÁ,,o de sua noÁ,,o de troca $\ddagger$ s que ocorrem em ambientes computacionais, e mesmo estendí-la a casos importantes n,o considerados explicitamente por Piaget. Para tanto, faz-se uma combinaÁ,,o dos conceitos presentes nos modelos b-sicos de troca computacional, definidos na seÁ,,o 4.1, com os conceitos extraldos da an lise dos processos de trocas em pequenos grupos (PIAGET, 1973b).

Assim, define-se troca em ambiente computacional como qualquer seq, Íncia de operaÁıes realizada por dois agentes, que tenha uma das formas b-sicas mostradas nos diagramas de seq, Íncias da Figura 2. Denomina-se de troca do tipo produtor-consumidor qualquer troca que siga o padr, ,O Prd-Cns da Figura 2, porque a seguinte interpretaÁ, „o È possìvel para a sua seq, Íncia de operaÁıes:
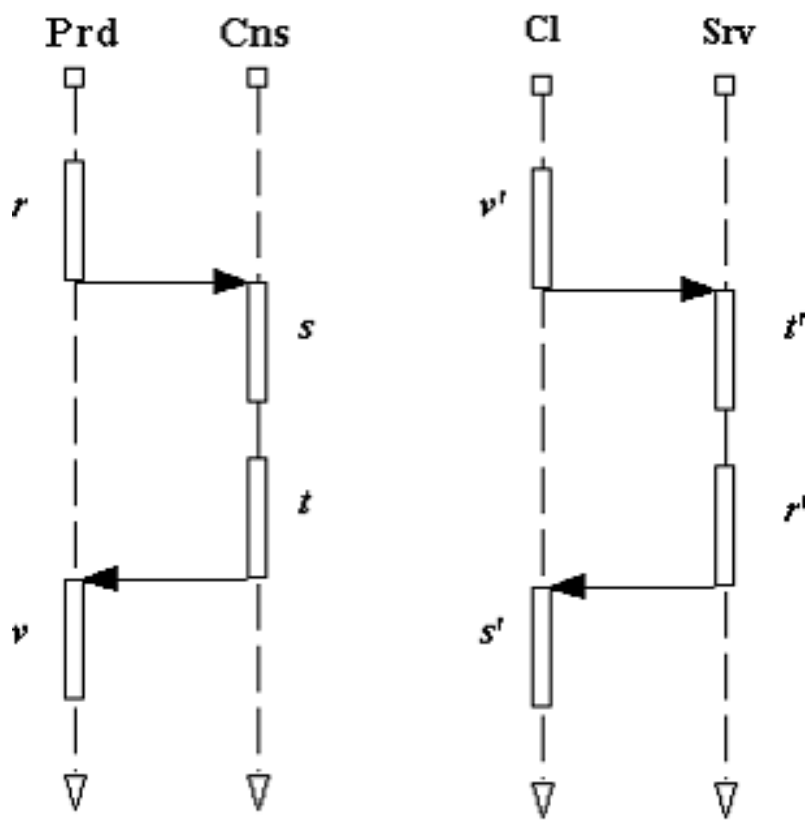

Figura 2 - Formas b·sicas de interaÁ,„o 
$\Sigma$ Prd $=$ Agente produtor

$\Sigma C n s=$ Agente consumidor

$\Sigma r=$ Valor de investimento para Prd da aÁ,o realizada por Prd

$\Sigma s=$ Valor de satisfação para Cns da aÁ,,o realizada por Prd

$\Sigma t=$ Valor de reconhecimento por parte de Cns da satisfaÁ,,o causada pela aÁ, o de Prd

$\Sigma v=$ Valor de acumulação de crÈdito por Prd em funÁ,,o do reconhecimento de Cns

Denomina-se troca do tipo cliente-servidor qualquer troca que siga o padr, ,o CnsSrv da Figura 2, porque a seguinte interpretaÁ,,o È possìvel para a sua seq,Íncia de operaÁıes:

$\Sigma C n s=$ Agente cliente

$\Sigma S r v=$ Agente servidor

$\Sigma V^{\prime}=$ Valor de crédito de Cns frente a

Srv devido a aÁıes anteriores realizadas por $\mathrm{Cns}$

$\Sigma t^{\prime}=$ Valor de reconhecimento de Srv do crÈdito pretendido por Cns

$\Sigma r^{\prime}=$ Valor de investimento de Srvem aÁ,,o para $C n s$

$\Sigma s^{\prime}=$ Valor de realização de crÈdito de Cns em funÁ,,O do investimento de Srv

Aos valores correspondentes a aÁıes efetivas dos agentes (valores $r, r^{\prime}, s$ e $s^{\prime}$ ), Piaget chamou de valores reais, porque dizem respeito $\ddagger$ valoraÁ,,o de ações concretas dos agentes. Os valores correspondentes a crËditos ou dĖbitos adquiridos ou reconhecidos (valores $v, v^{\prime}, t$ e $t^{\prime}$ ), chamou de valores virtuais, porque se referem a representações mentais que os agentes criam das situaÁıes de troca que se estabeleceram entre eles.

\subsection{As regras morais e jurídicas e o equi- líbrio das trocas sociais}

As regras sociais (normas, leis), na teoria sociolÛgica de Piaget, $\mathrm{s}, 0$ regras operatÛrias que tím por funÁ,,o garantir o equilìbrio das trocas realizadas pelos agentes em interaÁ,o.

Piaget considera que toda troca se realiza em duas etapas: a etapa da acumulação de valores virtuais e a etapa da realização de valores virtuais. Cada etapa fica definida por uma regra de equilíbrio especìfica, que Piaget formula em termos de variaÁ,o dos valores $r, s, t, v, r^{\prime}, s^{\prime}, t^{\prime}$ 'e $v^{\prime}$.

Piaget descreveu a etapa de acumulação de valores virtuais em termos que, relacionados ‡ Figura 2, s, o os de trocas do tipo produtor-consumidor. Ele denominou esse tipo de regra de tipo I. J. a etapa de realização de valores virtuais, ele descreveu em termos de trocas do tipo cliente-servidor. E denominou de regra de tipo //esse segundo tipo de regra.

Essas regras podem ser expressas como:

Regra de acumulação de valores virtuais:

$$
\downarrow(r)+\uparrow(s)+\downarrow(t)+\uparrow(v)=0
$$

\Regra de realização de valores virtuais:

$$
\downarrow\left(v^{\prime}\right)+\uparrow\left(t^{\prime}\right)+\downarrow\left(r^{\prime}\right)+\uparrow\left(s^{\prime}\right)=0
$$

onde as setas indicam variaÁıes qualitativas positivas $(\uparrow)$ e negativas $(\downarrow)$ dos valores em jogo.

A regra de acumulação de valores virtuais resume a idÈia de que, se o produtor Prd realizou uma aÁ,o com investimento $r$ 
de recursos e seu parceiro Cns deu-lhe o crËdito devido, ent, o $v=r$ È o crÈdito que Prd adquiriu frente a $C n s$, por ter realizado essa aÁ,,o para ele, e a igualdade representa que o crÈdito acumulado È equivalente ao investimento que ele realizou.

A regra de realização de valores virtuais resume a idÈia de que, se o cliente $\mathrm{Cl}$ tem um crÈdito $v$ ' por ter realizado uma aÁ,,o para o servidor Srv, que o servidor Srv reconhece esse dÈbito e compensa com uma aÁ, o r', ent, o s' = v'È a satisfaÁ,,o recebida por $C /$ em funÁ,,o da realizaÁ,,o de seu crÈdito, e a igualdade representa a realizaÁ,,o completa desse crÈdito.

Quando cada uma dessas regras È respeitada, diz-se que os valores são conservados, nas etapas respectivas. ${ }^{4}$. Um ciclo completo de interaÁ,,o, com o andamento das duas etapas em seq, Íncia, realizado nas seguintes condiÁıes:

¿cada agente sÛ cobra o crÈdito que ele efetivamente tem $\left(v^{\prime}=v\right)$ e cada agente paga exatamente o dÈbito que reconheceu $\left(t^{\prime}=t\right)$

¿cada etapa garante a conservaÁ,,o dos valores envolvidos, isto $\dot{E}$, as duas regras S,,O respeitadas,

tem como conseq, Íncia o conjunto de igualdades $v=r, v^{\prime}=v, s^{\prime}=v^{\prime}$, que implica $s^{\prime}=r$, isto $\dot{E}$, o agente que realizou a primeira aÁ,,o tem como resultado final um valor de satisfaÁ,,o equivalente ao seu valor de investimento inicial. Essa conservaÁ,o geral dos valores, no nlvel em que engloba as duas etapas seq, enciais, caracteriza o equilíbrio normativo do sistema.
Essa situaÁ,o favor·vel de equilìbrio normativo pode ser representada mais direta e significativamente atravĖs da adiÁ,,o termo a termo das duas regras de conservaÁ,o de valores (a de acumulaÁ,,o e a de realizaÁ,,o), combinado-as em uma 'nica regra. Fazendo isso, cancelando os valores virtuais complementares ( $\neq(v)$ com $\varnothing v i ́)$, e $\varnothing t$ ) com $\neq($ tí)), obtemos a regra:

$$
\begin{aligned}
& \downarrow(r)+\uparrow(s)+\downarrow(t)+\uparrow(v)=0 \\
& \frac{\downarrow\left(v^{\prime}\right)+\uparrow\left(t^{\prime}\right)+\downarrow\left(r^{\prime}\right) \uparrow\left(s^{\prime}\right)=0}{\downarrow(r)+\uparrow(s)+\downarrow\left(r^{\prime}\right)+\uparrow\left(s^{\prime}\right)=0}
\end{aligned}
$$

a qual expressa uma lei de equilìbrio que deixa de lado os valores virtuais e sû envolve valores reais.

Leis de equilìbrio que envolvem valores virtuais $\mathrm{S}$, ,O chamadas por Piaget de leis de equilíbrio moral e leis que envolvem somente valores reais $\mathrm{s}$, ,o chamadas de leis de equilíbrio econômico. O equilìbrio econÙmico pressupıe, portanto, o equilìbrio moral.

Essa estrutura normativa para sistemas computacionais, que foi introduzida em Costa (2002), pode ser aplicada a qualquer situaÁ,,o de interaÁ,,o na qual seja possìvel identificar os papÈis de cliente e servidor ou de produtore consumidor.

Como cada um desses papÈis pode ser identificado em praticamente todo tipo de sistema computacional onde haja interaÁıes (seja do sistema com seus usu.rios, seja de agentes do sistema entre si, seja dos usu.rios entre si), essa estrutura normativa tem praticamente aplicabilidade universal aos sistemas de computaÁ,,o.

4 Note-se que È possìvel que uma troca inicie pelo ciclo II, quando ent,,o o agente A, que a inicia no papel de cliente, adquire uma dìvida em relaÁ, o ao agente $B$ que lhe atende a requisiÁ,,o, dl̀vida que ser- paga atravĖs de um clico I, iniciado pelo prÛprio A, ou por um ciclo II, iniciado por B. 


\section{Sugestão de um modo de aplicação da teoria piagetiana dos valores de troca aos ambi- entes de ensino-aprendizagem}

A aplicaÁ,,o da teoria piagetiana dos valores de troca qualitativos aos ambientes de ensino-aprendizagem pressupı uma tomada de posiÁ,,o relativamente aos que tipos de trocas sociais que ocorrem nesses ambientes, desde o ponto de vista operacional.

Aqui, assumimos que $\mathrm{s}, \mathrm{O}$ dois os tipos principais de trocas sociais que ocorrem em um ambiente de ensino-aprendizagem. Por um lado, tem-se as produÁıes materiais dos professores e alunos envolvidos no ambiente. S,o os textos, figuras, animaÁıes, p·ginas, apresentaÁıes, vìdeos, programas, simulaÁıes e todo outro tipo de documento ativo ou passivo que os participantes do ambiente disponibilizam uns aos outros, em car-ter p blico ou privado.

O segundo tipo de trocas sociais que ocorrem em ambientes de ensino-aprendizagem $s,, 0$ as conversaÁıes, isto Ė, as trocas realizadas atravÈs de di·logos mediatos (atravÈs de correio eletrÙnico, chats, fûruns, videoconferíncias, etc.) ou imediatos (presenciais).

Consideramos que esses dois tipos de trocas $\mathrm{n}, \mathrm{O}$ s,, 0 independentes um do outro, nem iguais em import,ncia operacional. Consideramos que o tipo fundamental de trocas È o das trocas materiais, porque È ele que d. o sentido mesmo da formaÁ,,o do grupo social que participa do ambiente e È a raz,o de sua permaníncia no tempo, qual seja, a produÁ,,o material dos resultados de conhecimento que o grupo vai alcanÁando.
As conversaÁıes tím uma import,ncia operacional que È complementar a das trocas materiais e tím o sentido de instrumentos de coordenaÁ,,o das trocas.... pelas conversaÁıes que o grupo se organiza, reage a acontecimentos relevantes do processo de troca material, etc., mas È pelas aÁıes materiais que ele produz os resultados que s,,o a raz,,o de sua existíncia.

... claro que as conversaÁıes podem ser usadas como forma 'nica de troca, em um grupo orientado para a pura troca verbal de informaÁ,,o entre seus participantes. PorÈm, essa È uma situaÁ,o particular, dentro de um ambiente de ensino-aprendizagem. A situaÁ,,o geral È a de um grupo de alunos e professores que age em conjunto para ter produÁıes materiais como resultados finais de suas interaÁıes. Por isso, consideramos as trocas materiais como as trocas fundamentais.

Representamos nossa concepÁ,,o geral das trocas sociais em um ambiente computacional de ensino-aprendizagem atravĖs da Figura 3, que mostra os tipos de aÁıes sociais que podem ser realizadas por um grupo em um ambiente computacional. Essa figura, que È um refinamento da figura introduzida em Behar (1998), explicita a variedade das trocas sociais possìveis e se constitui em uma expans,,o do conhecido esquema $S$-O de Piaget.

Dada essa concepÁ,,o, assim como a metodologia de aplicaÁ,,o da teoria piagetiana das trocas sociais aos ambientes computacionais que expusemos anteriormente, toda a teoria se aplica diretamente aos ambientes computacionais de ensinoaprendizagem. 


\title{
teoria \& prática
}

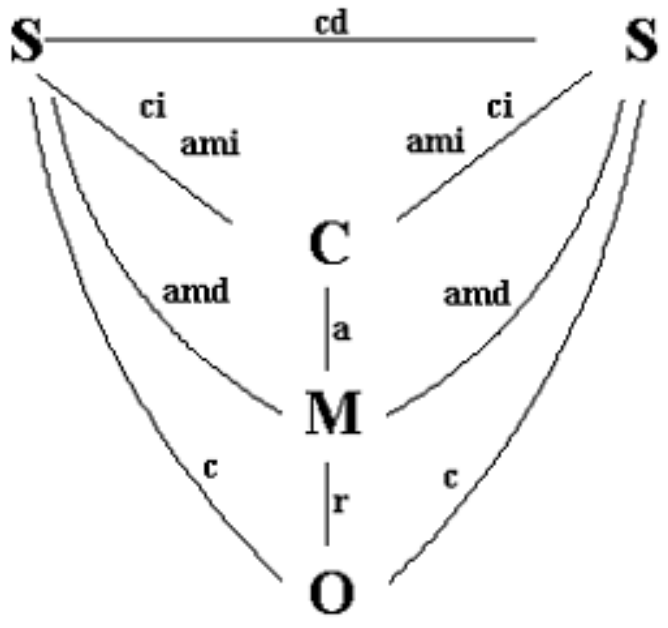

\author{
$\mathrm{S}=$ sujeito \\ $\mathrm{C}=$ computador \\ $\mathrm{M}=$ material produzido \\ $\mathrm{O}=$ objeto do conhecimento \\ cd = conversação direta \\ ci $=$ conversação indireta \\ $\mathrm{a}=$ armazenamento \\ ami $=$ ação material indireta \\ and = ação material direta \\ $\mathbf{r}=$ representação \\ $\mathbf{c}=$ conhecimento
}

Figura 3 - AÁıes sociais em ambientes computacionais de ensino-aprendizagem

... possìvel analisar, por exemplo, os comportamentos dos agentes em relaÁ,,O a regras normativas de car-ter geral (regras sociais v-lidas para todos os agentes) ou a regras normativas de car·ter particular (acordos locais entre agentes em interaÁ,o, estabelecidas por negociaÁıes entre eles), a an·lise do grau de cooperaÁ,o e de competiÁ,,o estabelecido entre os usu.rios do sistema, pela definiÁ, ,o dos valores que eles respectivamente atribuem $\neq s$ suas operaÁres de troca, assim como o modo com que seguem as leis de equilìbrio e realizam as operaÁıes de regulaÁ,o de suas trocas.

Outras questies, tais como as de interaÁıes individuais, intra-grupos e mesmo interaÁıes inter-grupos, etc., concretizam-se naturalmente no contexto daqueles ambientes, seguindo diretamente os prûprios mÈtodos de an-lise usados por Piaget, de modo que o problema de estudar todos esses aspectos morais e jurldicos dos ambientes computacionais de ensino-aprendizagem se torna agora, com a instrumentalizaÁ,,o fornecida aqui, um problema que pode ser enfrentado sistematicamente.

\section{Conclusão}

Este artigo $\mathrm{n}_{\text {, }} \mathrm{o}$ dispensa a leitura dos textos piagetianos previamente $\ddagger$ aplicaÁ, ,o da teoria dos valores de troca $\ddagger$ an lise de ambientes de ensino-aprendizagem. Ao contr rio, trata-se de um complemento para aquela leitura.

Nesse sentido, apresentamos apenas uma articulaÁ,,o dos conceitos piagetianos de valor de troca e de regra normativa, com os conceitos de sistemas cliente-servidor e produtor-consumidor, presentes em qualquer ambiente computacional e de ensino-aprendizagem. Consideramosque essa articulaÁ,,o, introduzida em Costa (2002), È a chave para a aplicaÁ, ,o dessa teoria a esses sistemas. 
Em Rodrigues (2003a) apresentamos uma proposta de aplicaÁ,,o da teoria dos valores de troca a sistemas multiagentes, enquanto que em Rodrigues (2003b) apresentamos uma demonstraÁ,,o do uso dessa aplicaÁ,o na simulaÁ,,o de processos sociais.
Uma primeira tentativa de an-lise das conversaÁıes do ponto de vista dos valores de trocas que elas envolvem pode ser encontrada em Ribeiro (2003), que analisa interaÁıes em chats.

\section{Referíncias}

BEHAR, P. Análise Operatória de Ferramentas Computacionais de Uso Individual e Coletivo. Porto Alegre: CPGCC/UFRGS, 1998. (Tese. Doutorado).

COSTA, A. C. da R.; DIMURO, G. P. Uma Estrutura Formal Normativa para Sistemas Computacionais. In: Oficina de InteligÍncia Artificial, 6., Pelotas. Anais... Pelotas: UCPel, 2002, p.77-83. Disponìvel em: <http://gmc.ucpel.tche.br/valores>

PIAGET, J. Ensaio sobre a Teoria das Valores Qualitativos em Sociologia Est·tica (îSincrÙnicaî). PIAGET, J. In: Estudos Sociológicos. S.I : s.e., 1973a. P.114-163.

Études Sociologiques. GenËve: Droz, 1965.

. Estudos Sociológicos. Rio de Janeiro: Forense, 1973b.

. A explicaÁ,o em sociologia. PIAGET, J. Estudos Sociológicos. 1973c. P.17-113.

Genetic logic and sociology. PIAGET, J. Sociological Studies. 1995. P.184-214.

Introduction a l'Épistémolgie Génétique. Paris: Presses Universitaires de France, 1950.

Ensaio de Lógica Operatória. Porto Alegre: Globo, 1973d.

__. As operaÁıes IÛgicas e a vida social. PIAGET, J. Estudos Sociológicos. 1973e. P.164-196.

__. As relaÁıes entre a moral e o direito. PIAGET, J. Estudos Sociológicos. 1973f. P.197-231.

Sociological Studies. London: Routlege, 1995.

Ensaio sobre a teoria dos valores qualitativos em sociologia est.tica (îSincrÙnicaî). PIAGET, J. In: Estudos Sociológicos. Rio de Janeiro: Forense, 1973b P.114-163.

RIBEIRO, L. O. Evidências de Reciprocidade Normativa num Ambiente Virtual de Aprendizagem na Formação de Professores para EAD. Porto Alegre: PGIE/UFRGS, 2003. (Trabalho da Disciplina de Inteligíncia Artificial Construtivista 2002/2) Disponìvel em:<http://gmc.ucpel.tche.br/valores>.

RODRIGUES, M. R.: COSTA, A . C. da R.; BORDINI, R. H. A system of eschange values to support social interactions in artificial societies. In: Autonomous Agentes and Multiagent Systems AAMAS 2003, Melbourne, July 2003a.

RODRIGUES, M. R.; COSTA, A . C. da R. Using qualitative exchange values to improve the modelling of social interactions. In: Multiagent Based Simulation - MABS 2003, Melbourne, July 2003b. 


\section{AntÙnio Carlos da Rocha Costa}

Engenheiro, mestre em CiÍncia da ComputaÁ,o (UFRGS), doutor em Ciĺncia da ComputaÁ,o (UFRGS), professor da Escola de Inform·tica (UCPel), orientador colaborador do PGIE/UFRGS e do PPGC/UFRGS.

E-mail: rocha@atlas.ucpel.tche.br 